\title{
Aplicación de modelos cinéticos en la cementación de oro con cinc desde soluciones con tiosulfato y amoniaco
}

\author{
P. Navarro ${ }^{a *}$, C. Vargas ${ }^{*}$, F.J. Alguacil ${ }^{* *}$ y R. Alvarez ${ }^{* a}$
}

Resumen La lixiviación de oro en medio tiosulfato es una de las alternativas propuestas a la lixiviación en medio cianuro, especialmente cuando se consideran algunas menas auríferas. A su vez, se han considerado distintas tecnologías para la recuperación del metal de las disoluciones de lixiviación y, de estas, la cementación con cinc es una de las menos estudiadas. En el presente trabajo, se han aplicado distintos modelos cinéticos a los resultados experimentales de cementación de oro con cinc en polvo, siendo el modelo cinético de pasivación el que mejor ajusta estos. Se considera que la cementación del oro responde a un régimen de control difusivo $(15,4 \mathrm{~kJ} / \mathrm{mol})$, estando favorecida la cementación del metal precioso al emplear mayores temperaturas o valores de $\mathrm{pH} /$ concentración de amoniaco en el medio acuoso.

Palabras clave Oro. Cinc. Cementación. Tiosulfato. Amoniaco. Cinética.

\section{Application of kinetics models on gold cementation by zinc from tiosulphate and ammonia solutions}

\begin{abstract}
Being thiosulphate leaching of gold one of the proposed alternatives to conventional cyanide leaching of several gold ores, different approaches for metal recovery from pregnant thisulphate solutions had been considered and from these, gold cementation by zinc had not been widely study. In the present work, several kinetics models had been tested on experimental results of gold cementation by powdered zinc, and under varying conditions results are best fitted to the passivation model. Overall, the cementation of the precious metal by zinc from ammoniacal thiosulphate solutions was concluded to be under mass transfer control, higher temperatures and $\mathrm{pH} /$ ammonia concentration were established to positively affect the rate of gold cementation.
\end{abstract}

Keywords Gold. Zinc. Cementation. Tiosulphate. Ammonia. Kinetics.

\section{INTRODUCCIÓN}

Aunque la lixiviación con cianuro es el método más comúnmente usado para la recuperación de oro de las distintas materias primas que lo contienen $^{[1]}$, algunos problemas en la utilización de este reactivo, entre otros, el carácter evidentemente tóxico de este agente de lixiviación, ha hecho que se consideren otros medios de lixiviación (Tabla I) como alternativas menos tóxicas a la utilización del cianuro en la hidrometalurgia del oro. Entre estas alternativas, el uso del tiosulfato en medio
Tabla I. Constantes de estabilidad de algunos complejos de oro(l) ${ }^{[2]}$

Table I. Stability constants for selected gold complexes ${ }^{[2]}$

\begin{tabular}{lcc}
\hline Especie & Log $\mathrm{K}$ & Estructura \\
\hline $\mathrm{Au}(\mathrm{CN})_{2}{ }^{-}$ & 38,3 & lineal \\
$\mathrm{Au}(\mathrm{SCN})_{2}^{-}$ & 17,0 & lineal \\
$\mathrm{AuTU}{ }_{2}^{2+}$ & 23,3 & lineal \\
$\mathrm{Au}\left(\mathrm{NH}_{3}\right)_{2}{ }^{+}$ & 26,0 & lineal \\
$\mathrm{Au}\left(\mathrm{S}_{2} \mathrm{O}_{3}\right)_{2}{ }^{3-}$ & 28,0 & lineal \\
\hline
\end{tabular}

(*a) Departamento de Ingeniería Metalúrgica, Facultad de Ingeniería, Universidad de Santiago de Chile, Casilla 10233, Santiago, Chile. E-mail: pnavarro@lauca.usach.cl

(**b) Centro Nacional de Investigaciones Metalúrgicas (CSIC), Avda. Gregorio del Amo 8, Ciudad Universitaria, 28040 Madrid, España. 
amoniacal y en presencia de cobre(II) parece que está tomando un renovado interés para su utiliza-

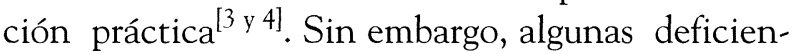
cias en los procesos de recuperación de oro en medio tiosulfato-amoniacal hacen que el proceso, hasta la fecha, no sea competitivo desde un punto de vista económico, si se compara con la lixiviación cianurante y los procesos asociados con la recuperación de oro de este medio.

Sin embargo, muchos de los procesos empleados en medio cianuro, se están aplicando a la recuperación de oro en medio tiosulfato, aunque con un grado de éxito variable. Entre estos procesos, la cementación ha alcanzado un relativo interés ${ }^{[3-7]}$, existiendo algunos problemas sin resolver en relación con la química del proceso y el mecanismo de reacción. Este estudio se ha llevado a cabo con el fin de intentar resolver algunas de estas lagunas e intentar ayudar a comprender el mecanismo de cementación del oro empleando cinc como agente cementante.

\section{MATERIALES Y PROCEDIMIENTO EXPERIMEN- TAL}

Los ensayos de cementación se llevaron a cabo en un reactor de vidrio de 0,751 de capacidad que se situó en un baño de agua termostatizado. El sistema se agitó de forma mecánica a 300 rpm y se midieron de forma continua las siguientes variables: temperatura, $\mathrm{pH}$ y potencial redox ( $\mathrm{pH}$-metro HANNA 8417 provisto de un electrodo combinado) y contenido de oxígeno en la disolución. Los ensayos se realizaron mediante la introducción de la disolución en el reactor y el borboteo de $\mathrm{N}_{2}$ al sistema hasta que el contenido de oxígeno en la disolución fue de 0,001 g/l, introduciéndose en este momento el polvo de cinc en el reactor y continuando el borboteo de nitrógeno a lo largo de todo el experimento. Las disoluciones de oro se prepararon a partir de $\mathrm{Na}_{3} \mathrm{Au}\left(\mathrm{S}_{2} \mathrm{O}_{3}\right)_{2} \cdot 2 \mathrm{H}_{2} \mathrm{O}$ (Alfaaesar) disuelto en agua destilada y adición de los otros componentes (grado RA) de la disolución hasta alcanzar las concentraciones requeridas para cada caso. El contenido de oro en la disolución se determinó mediante espectrofotometria de absorción atómica. El polvo de cinc empleado en el presente estudio presentaba un tamaño de partícula medio de $2,8 \mu \mathrm{m}$.

\section{RESULTADOS Y DISCUSIÓN}

Se ha llevado a cabo la modelación cinética de la cementación de oro mediante cinc desde disolu- ciones acuosas con tiosulfato-amoniaco asumiendo diferentes ecuaciones o modelos. Se supuso que las etapas del mecanismo de reacción son las siguientes:

- transporte de masa de reactantes desde una fase líquida a la interfase líquido-sólido de la reacción,

- reacción química en la interfase,

- transporte de masa de productos fuera de la interfase.

Para lo anterior se asume que la reacción química en la superficie de las particulas de cinc es irreversible y que las concentraciones de equilibrio en la superficie de reacción son muy pequeñas, con lo cual el tercer paso no sería una etapa limitante en la velocidad del proceso.

Los modelos aplicados a los resultados experimentales fueron:

- Modelo de cinética lineal de primer orden. En este caso, la ecuación cinética responde a la expresión

$$
\ln \frac{[\mathrm{A} u]_{t}}{[\mathrm{~A} u]_{0}}=-k_{1} t
$$

- Modelo de pasivación o inhibición. En este modelo, la ecuación es

$$
\ln \frac{[\mathrm{A} u]_{t}}{[\mathrm{~A} u]_{0}}=-k_{1}\left(\frac{\mathrm{A}}{\mathrm{V}}\right) t+\left(\frac{k_{1} k_{2}}{2 \mathrm{~V}}\right) t^{2}
$$

en la que el primer término contiene la constante cinética de primer orden, $\mathrm{k}_{1}$, que puede ser tanto una constante química como difusional dependiendo del mecanismo que controle al proceso de cementación. Este término es el que domina la cinética global del proceso, siendo el segundo término de la ecuación, que también contiene la constante de primer orden, una corrección al comportamiento no lineal del sistema. Se considera que las desviaciones observadas al comportamiento lineal, (Ec. (1)) se deben a la formación, más o menos progresiva, de un compuesto de caracter pasivante sobre la superficie del agente cementante de forma que, con el tiempo, disminuye el área sobre la que tiene lugar el proceso de cementación.

- Modelo cinético en condiciones de equilibrio. En algunos casos, por ejemplo cuando el sistema presenta sales complejas, la desviación del 
comportamiento lineal se atribuye a una condición en la que este se está aproximando al equilibrio de cementación, y la ecuación cinética correspondiente responde a la ecuación

$$
\ln \left(\frac{[\mathrm{Au}]_{t}-[\mathrm{Au}]_{e}}{[\mathrm{Au}]_{0}-[\mathrm{Au}]_{e}}\right)=-k_{1} t
$$

Se considera que en la cementación de oro con cobre, el valor de la concentración de oro en equilibrio es del orden de 0,007 mg/l. Se han evaluado los tres modelos cinéticos arriba descritos bajo una serie de condiciones experimentales, encontrándose que el modelo que mejor representa la cinética de cementación de oro con cinc desde disoluciones con tiosulfato y amoniaco es el modelo de inhibición o pasivación. Los valores encontrados para las constantes cinéticas de la correspondiente ecuación se muestran el la tabla II.

Aunque se observa que, en general, la cinética de cementación del oro está favorecida para aquellos casos en los que la relación $\left[\mathrm{NH}_{3}\right] /\left[\mathrm{S}_{2} \mathrm{O}_{3}{ }^{2-}\right]$ en el medio acuoso es más alta, otras condiciones que pueden afectar a este proceso de cementación se considerarán a continuación.

El efecto de la temperatura sobre la cementación del oro se estudió empleando una fase acuosa (500 $\mathrm{ml}, \mathrm{pH}$ 9,7) que contenia $0,008 \mathrm{~g} / \mathrm{l}$ de oro, $0,05 \mathrm{M}$

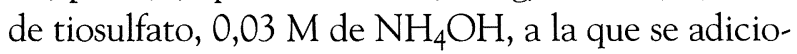
nó $1 \mathrm{~g}$ de cinc en polvo. Al analizar el efecto de la temperatura sobre la cementación del oro (Fig. 1) se observa que esta variable afecta de alguna forma a la

Tabla II. Constantes cinéticas en la cementación de oro con cinc

Table II. Kinetics constants in the gold cementation with zinc

\begin{tabular}{ccccc}
\hline pH & $\begin{array}{c}{\left[\mathrm{S}_{2} \mathrm{O}_{3}^{2-}\right],} \\
\mathbf{m o l} / \mathrm{L}\end{array}$ & $\begin{array}{c}{\left[\mathrm{NH}_{3}\right] /} \\
{\left[\mathrm{S}_{2} \mathrm{O}_{3}^{2-}\right]}\end{array}$ & $\mathrm{K}_{1}, \mathrm{~cm} / \mathrm{min}$ & $\mathrm{K}_{2}, \mathrm{~cm} / \mathrm{min}$ \\
\hline 9,0 & 0,05 & 3,0 & 0,116 & 3425 \\
9,0 & 0,05 & 1,8 & 0,033 & 1073 \\
9,0 & 0,2 & 0,5 & 0,003 & 264 \\
9,0 & 0,2 & 0,2 & 0,002 & 339 \\
9,7 & 0,05 & 1,8 & 0,001 & 169 \\
9,7 & 0,4 & 0,1 & $<0,001$ & 196 \\
10,5 & 0,05 & 1,8 & 0,105 & 1743 \\
10,5 & 0,05 & 0,6 & 0,008 & 183 \\
10,5 & 0,2 & 0,2 & 0,004 & 236 \\
\hline
\end{tabular}

Concentración de oro en la disolución: 0,008 $\mathrm{mg} / \mathrm{L}$ Volumen de disolución: $500 \mathrm{~mL}$. Agente cementante: $1 \mathrm{~g}$ cinc en polvo. Temperatura: $20^{\circ} \mathrm{C}$. cementación del metal precioso, especialmente en los tiempos más cortos, si bien esta influencia se minimiza para tiempos de reacción más prolongados, por ejemplo, $30 \mathrm{~min}$, donde el rendimiento de la reacción de cementación es cercano al 90 \% para los tres casos considerados. Esta influencia (positiva) sobre la cementación del oro se puede asociar a la disminución de la viscosidad de la disolución al aumentar la temperatura, de forma que el (los) complejo(s) de oro se mueven de forma más libre a través del medio acuoso. Con objeto de calcular la energía de activación del proceso, se realizó un ajuste de los datos con el modelo cinético de inhibición; puesto que en este modelo el único elemento variante en el primer término de la ecuación es $\mathrm{k}_{1}$, su dependencia de la temperatura puede ser descrita por la ecuación de Arrhenius. La energia de activación calculada a partir de la representación de $-\ln \mathrm{k}_{1}$ frente a $1 / \mathrm{T}$ resulto ser de $15,4 \mathrm{~kJ} / \mathrm{mol}$, por lo que se puede considerar que la cementación del oro se situa bajo un régimen de control difusivo.

Se ha observado (Tabla II) que para un mismo valor del $\mathrm{pH}$, el aumento de la relación amoniaco/tiosulfato en la disolución acuosa favorece la cementación del oro. Por otro lado, para condiciones experimentales similares (disolución $(500 \mathrm{ml}$ ) conteniendo $0,008 \mathrm{~g} / \mathrm{l} \mathrm{Au}, 0,05 \mathrm{M}$ tiosulfato y $0,09 \mathrm{M} \mathrm{NH}_{4} \mathrm{OH}$ y a la que se adicionó $1 \mathrm{~g}$ de cinc), al aumentar el $\mathrm{pH}$ del medio acuoso, se tiende a favorecer la cementación de este metal (Fig. 2). Este hecho se podría relacionar a que, al aumentar el $\mathrm{pH} /$ concentración de amoniaco en el

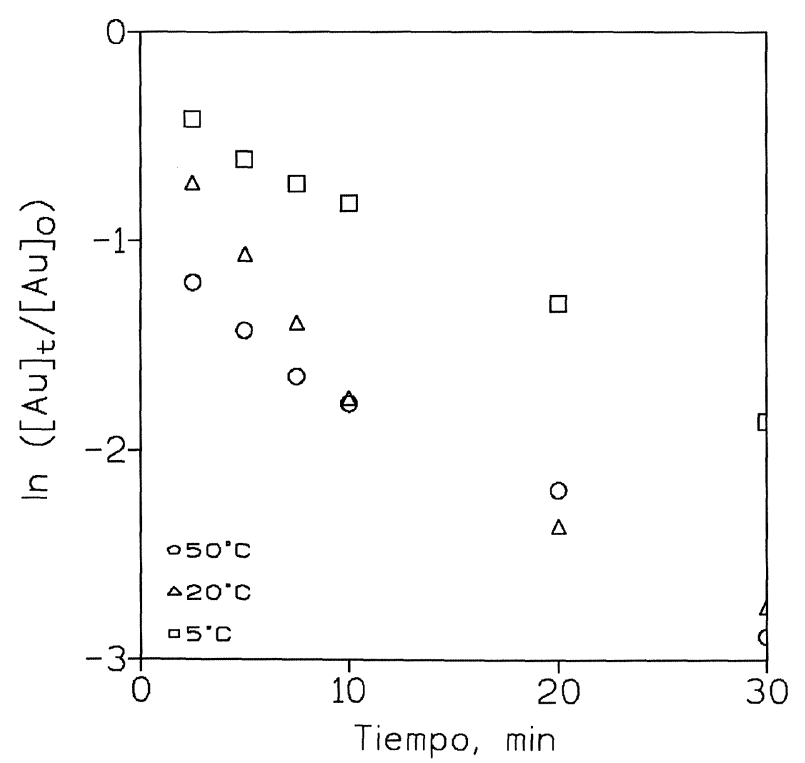

Figura 1. Influencia de la temperatura sobre la cementación de oro.

Figure 1. Influence of temperature on gold cementation. 
Aplicación de modelos cinéticos en la cementación de oro con cinc desde soluciones con tiosulfato y amoniaco

P. Navarro, C. Vargas, F.J. Alguacil y R. Alvarez

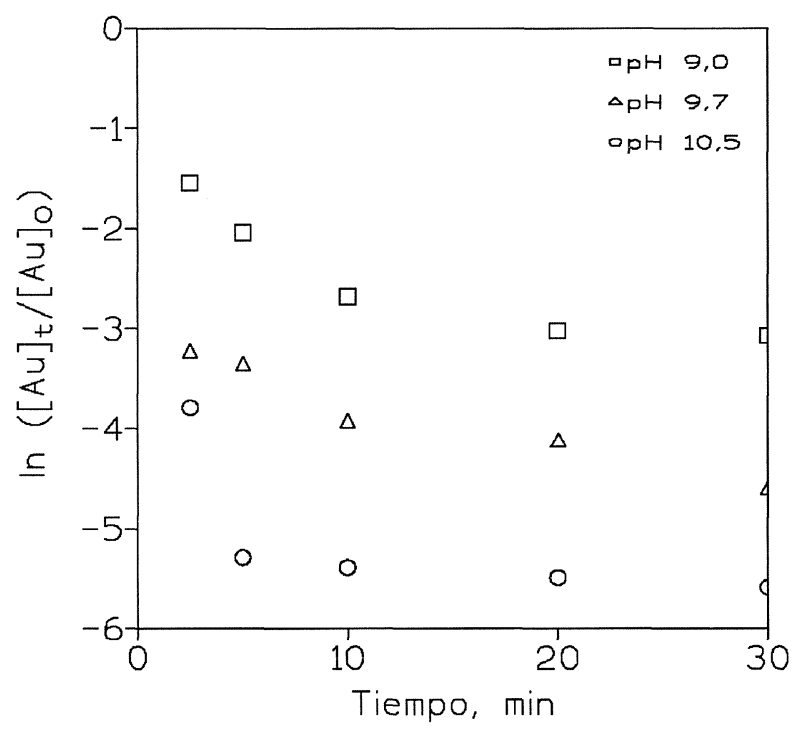

Figura 2. Influencia del pH sobre la cementación de oro. Temperatura $25^{\circ} \mathrm{C}$

Figure 2. Influence of $\mathrm{pH}$ on gold cementation. Temperature $25^{\circ} \mathrm{C}$.

medio, una parte del oro podría existir como el complejo diaminoro(I), más pequeño que el di(tiosulfato) aurato(I), y por lo tanto con una mayor movilidad a través del medio. Sin embargo, la existencia del complejo amoniacal, en un medio tiosulfato-amoniacal, está bajo una cierta controversia, ya que en algunos casos se describe que la formación de esta especie no está favorecida, y solo el complejo oro-tiosulfato existe en el medio acuoso $^{[4}$ y 8$]$; sin embargo, también se menciona que en estos sistemas mixtos amoniaco-tiosulfato es posible la presencia de complejos mixtos del oro(I) con los dos ligandos ${ }^{[9]}$. Teniendo en cuenta el efecto favorable (hasta ciertos límites) que el amoniaco tiene sobre la cementación del oro, es indudable que existe una necesidad de mayor información sobre el sistema $\mathrm{Au}-\mathrm{NH}_{3}-\mathrm{S}_{2} \mathrm{O}_{3}{ }^{2-}$.

El efecto de la concentración de oro sobre la cementación de este metal se muestra en la figura 3. Las condiciones experimentales fuerón: $500 \mathrm{ml}$ de disolución a $\mathrm{pH}$ 9,7, conteniendo oro, 0,05 $\mathrm{M}$ de tiosulfato y $0,09 \mathrm{M}_{\text {de }} \mathrm{NH}_{4} \mathrm{OH}$. Se observa que la cinética de cementación del metal precioso está más favorecida al emplear la disolución de oro más diluida, aunque para tiempos de reacción largos, por ejemplo, $20 \mathrm{~min}$, el rendimiento de la operación se acerca al $95 \%$ en los dos casos considerados.

En cuanto a la evaluación de la influencia de la cantidad de agente cementante añadida al sistema sobre la cementación del oro, los resultados obtenidos se muestran en la tabla III.

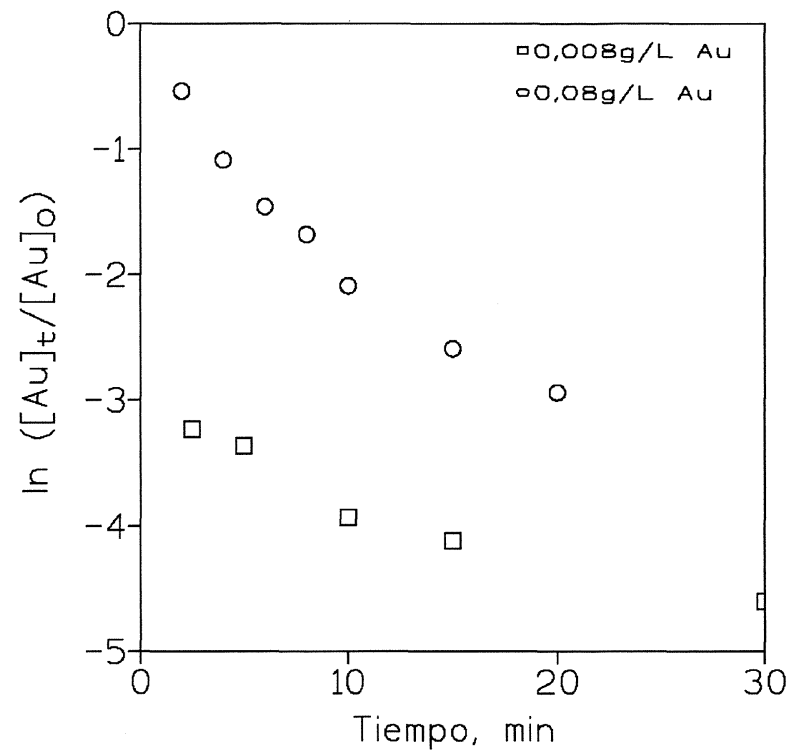

Figura 3. Influencia de la concentración inicial de oro en la cementación del metal. Temperatura $25^{\circ} \mathrm{C}$.

Figure 3. Influence of intial gold concentration on metal cementation. Temperature $25^{\circ} \mathrm{C}$.

Tabla III. Influencia de la cantidad de polvo de cinc adicionada al sistema sobre la cementación de oro

Table III. Influence of the added zinc dust on gold cementation

\begin{tabular}{ccc}
\hline $\begin{array}{c}\text { Cinc añadido } \\
\mathbf{g}\end{array}$ & $\begin{array}{c}\text { \% cementación } \\
(\mathbf{2}, \mathbf{5} \mathbf{~ m i n})\end{array}$ & $\begin{array}{c}\text { \% cementación, } \\
(\mathbf{2 0} \mathbf{~ m i n})\end{array}$ \\
\hline 0,1 & 53 & 94 \\
0,25 & 78 & 91 \\
1 & 92 & 90 \\
\hline
\end{tabular}

Volumen de disolución: $500 \mathrm{~mL}$. Concentración de oro: 0,008 $\mathrm{mg} / \mathrm{L}$. Concentración de tiosulfato: 0,05 M. Concentración de $\mathrm{NH}_{4} \mathrm{OH}: 0,09$ M. pH de la disolución: 9,7. Temperatura: $20^{\circ} \mathrm{C}$.

Se puede observar que cuando aumenta la cantidad de polvo de cinc añadida al sistema, la recuperación de oro es prácticamente la misma ( 20 $\mathrm{min})$, sin embargo, para tiempos cortos (2,5 $\mathrm{min})$ la cinética de cementación de oro se ve favorecida al aumentar esta cantidad.

La adición de cinc al sistema como cinc(II) (sulfato) tiene un efecto negativo sobre la cementación del oro. Los iones $\mathrm{Zn}^{2+}$ en medio amoniacal existen de forma predominante como el complejo $\mathrm{Zn}\left(\mathrm{NH}_{3}\right)_{4}{ }^{2+}$, con lo que "roban" amoniaco del sistema y, como ya se discutió anteriormente, la disminución de la concentración de amoniaco libre en la disolución afecta de forma negativa a la cementación del oro. Otra reacción 
secundaria debida a la presencia de $\mathrm{Zn}^{2+}$, y que afecta de forma negativa al proceso de cementación, sería la precipitación de alguna especie sobre la superficie cementante con la consiguiente pasivación de la misma. No se han considerado, sin embargo, las posibles reacciones del cinc(II) con el ion tiosulfato debido a las constantes de estabilidad bajas de dichas especies ${ }^{[10}$ y 11$]$.

\section{CONCLUSIONES}

La cementación de oro, de disoluciones que contienen tiosulfato y amoniaco, mediante cinc puede ser un método efectivo para la recuperación de este metal precioso de estas disoluciones. En general, un aumento de la temperatura y un aumento de la relación $\left[\mathrm{NH}_{3}\right] /$ tiosulfato en el sistema favorece el proceso de cementación, mientras que la presencia de cinc(II) en el medio tiende a inhibir este proceso, especialmente en los primeros momentos del mismo. El proceso global de cementación responde a un control difusivo, mientras que los resultados experimentales parecen responder bien al modelo de pasivación.

\section{NOMENCLATURA}

$[\mathrm{Au}]_{0},[\mathrm{Au}]_{\mathrm{t}},[\mathrm{Au}]_{\mathrm{e}}$, : concentraciones de oro inicial, a un tiempo $t$ y en condiciones de equilibrio, respectivamente. $\mathrm{k}_{1}, \mathrm{k}_{2}$ : constantes de reacción. A: área superficial del agente cementante. $\mathrm{V}$ : volumen de la disolución en el reactor. t: tiempo.

\section{Agradecimientos}

Los autores agradecen al CSIC (España) y a la USACH (Chile) la ayuda prestada mediante la concesión del Proyecto 2003CL0001. Los autores también agradecen a CONICYT (Chile), que gracias a los aportes a través del Proyecto FONDECYT $N^{\circ} 1020017$, han hecho posible su realización.

\section{REFERENCIAS}

[1] J. MARSDEN y I. House, The Chemistry of Gold Extraction, Ellis Horwood, London, United Kingdom, 1992.

[2] P. Navarro, C. Vargas, A. Villarroel y FJ. Alguacil, Hydrometallurgy 65 (2002) 37-42.

[3] M.G. Aylmore y D.M. Muir, Min. Eng. 14 (2001) 135 174.

[4] A.C. Grosse, G.W. Dicinoski, M.J. Shaw y P.R. HADDAD, Hydrometallurgy 69 (2003) 1-21.

[5] E. Guerra y D. Dreisinger, Hydrometallurgy 51 (1999) 155-172.

[6] J. Sedzimir, Hydrometallurgy 58 (2000) 175-178.

[7] J.B. HiSKEY Y J. LEE, Hydrometallurgy 69 (2003) 45-56.

[8] E. Molleman y D.B. Dreisinger, Hydrometallurgy 66 (2002) 1-21.

[9] P.L. Breuer y M.I. JefFrey, Hydrometallurgy 65 (2002) 145-157.

[10] D. Feng and J.S.J. VAn DeVenter, Hydrometallurgy 64 (2002) 231-246.

[11] C. Vargas, G. Cifuentes, P. Navarro y P. Orrego, Rev. Metal. Madrid 40 (2004) 101-108. 